\title{
Philosophiques
}

\section{Réponse à Michel Ratté}

\section{Rainer Rochlitz}

Volume 27, numéro 2, automne 2000

URI : https://id.erudit.org/iderudit/004977ar

DOI : https://doi.org/10.7202/004977ar

Aller au sommaire du numéro

Éditeur(s)

Société de philosophie du Québec

ISSN

0316-2923 (imprimé)

1492-1391 (numérique)

Découvrir la revue

Citer cet article

Rochlitz, R. (2000). Réponse à Michel Ratté. Philosophiques, 27(2), 443-448.

https://doi.org/10.7202/004977ar

Ce document est protégé par la loi sur le droit d'auteur. L'utilisation des services d'Érudit (y compris la reproduction) est assujettie à sa politique d'utilisation que vous pouvez consulter en ligne.

https://apropos.erudit.org/fr/usagers/politique-dutilisation/
Cet article est diffusé et préservé par Érudit.

Érudit est un consortium interuniversitaire sans but lucratif composé de l’Université de Montréal, l'Université Laval et l'Université du Québec à Montréal. Il a pour mission la promotion et la valorisation de la recherche. https://www.erudit.org/fr/ 


\title{
Réponse à Michel Ratté
}

\author{
RAINER ROCHLITZ \\ CNRS \\ rainer.rochlitz@
}

Mon ami Michel Ratté cherche à pointer les failles et les faiblesses de mes propositions esthétiques. S'il avait raison, ce qu'il appelle, de façon assez flatteuse, "la position la plus exigeante dans le débat des théoriciens de l'esthétique et de l'art en France » ne tiendrait donc finalement guère ses promesses. On devrait dès lors se demander si l'esthétique française contemporaine, selon lui dans l'ensemble moins " exigeante » que la mienne, n'est pas dans un piteux état. Je ne pourrai évidemment parler que pour moimême.

Parmi les nombreux points litigieux relevés, ma réponse forcément brève et tout aussi inévitablement apologétique ne pourra retenir qu'un petit nombre. Ce sera : 1 . la question de l'adéquation des jugements de fait aux œuvres, liée par Michel Ratté à celle de "l'expressivité » ou encore de l'opacité de l'œuvre ; 2 . celle des espoirs fondés sur la critique, liée au rapprochement entre débat critique et débat sur les normes; et 3 . celle de "l'homologation » des œuvres, en rapport avec le caractère intersubjectif de l'art. Avant d'aborder ces différents points, il me faut cependant dire un mot sur mes choix philosophiques.

À plusieurs reprises, Michel Ratté souligne que je critique la " philosophie de la conscience », appelée aussi "philosophie du sujet » et que j'adhère à ce qu'il dénonce comme la « théorie consensuelle de la vérité » Ce choix lui semble être à l'origine de certaines des faiblesses qu'il me reproche. Il n'est guère possible de discuter ici ces problèmes complexes. Mais j'aimerais lui signaler qu'il existe de fortes raisons de vouloir échapper à certaines impasses conceptuelles qui résultent des aiguillages de la philosophie du sujet, telle qu'elle s'est développée de Descartes à Husserl et ses disciples. Elle s'est révélée incapable de rendre compte des phénomènes intersubjectifs, notamment sociaux, auxquels nous avons affaire, entre autres dans le domaine de l'art. Quant à la théorie de la vérité à laquelle il fait allusion dans des termes ambigus, elle repose sur l'idée que la « correspondance » entre les faits et nos affirmations - seule alternative sérieuse à la conception argumentée de la vérité - suggère quelque chose comme des "faits » non interprétés auxquels nous pourrions comparer nos affirmations. Même s'il existe toujours des défenseurs de la théorie de la vérité-correspondance, leur point de vue est aujourd'hui assez largement contesté. Car cette approche n'est en fait efficace que lorsque l'objet du litige s'offre à l'observation, situation qui suscite rarement des interrogations sur la vérité. 
1.

Michel Ratté observe que j'établis une relation entre notre connaissance descriptive des œuvres d'art et nos jugements esthétiques. Il appelle cette relation " dialectique », ce qui n'est guère gênant mais, à mon avis, n'apporte à l'idée de " lien » que des connotations qui risquent d'induire en erreur. Dans la mesure où le jugement répond, chez moi, à une "sollicitation fondamentale de l'œuvre », Ratté ajoute [p. 433] que je « semble faire pencher tout le problème de la liaison interne de l'évaluation et de la compréhension vers la question de l'adéquation possible avec le symbole artistique lui-même, l'œuvre " - Dans un second temps, il lie cette question à une "élucidation de l'expressivité même de cette sollicitation ", qui lui semble poser le problème de « l'opacité expressive de l'œuvre » [p. 434]. J'aimerais dissocier ces deux questions.

a) Du fait du primat du jugement de fait, Michel Ratté craint que « toutes les évaluations perdent leurs prétentions expressives en se dissolvant dans le jugement de fait le plus adéquat » [ibid.]. Il redoute donc que le fait de privilégier l'analyse la plus apte à rendre compte d'une œuvre nous conduise à sacrifier la pluralité des relations " expressives » avec les œuvres, autrement dit, si j'ai bien compris, la richesse des investissements subjectifs très divers qui se projettent sur les œuvres. Je ne crois pas que cette crainte soit justifiée. Car, d'une part, si nos débats critiques sur les œuvres tendent vers une perception juste, ils ne font pas disparaître la diversité des lectures, car il est rarement possible d'affirmer qu'une lecture ne découvre rien dans une œuvre ; son apport, et en ce sens un certain " relativisme ", sera donc conservé. Mais surtout, les jugements de fait ne sont jamais, selon moi, dissociables des hypothèses évaluatives sur la réussite et l'importance d'une œuvre. Autrement dit, l'intérêt de tout jugement de fait dépend de sa contribution à une lecture qui rende justice, autant que possible, à la qualité de l'œuvre, ou qui lui donne toutes ses chances. Il n'y a donc pas, chez moi, de primat indépendant des jugements de fait à propos des œuvres, et le reproche de Michel Ratté est infondé. Ma distinction entre les œuvres et leurs supports matériels, les " habitacles ", me conduit à cette conclusion.

b) Le terme expressivité ne fait pas partie de mon vocabulaire, mais de celui de Michel Ratté, et il me semble peu clair. Quant au lien entre " l'adéquation » du jugement et " l'expressivité » de la sollicitation de l'œuvre, je ne suis pas sûr de comprendre la remarque. Quoi qu'il en soit, ce sur quoi Ratté souhaite insister, c'est de toute évidence "l'opacité » de l'œuvre, autrement dit ce qui en elle résiste à toute prétention à une connaissance adéquate. Ratté souligne [p. 435] que je n'aborde pas cette question. C'est que je ne suis pas sûr qu'elle soit féconde, pour au moins deux raisons. i) Il est clair que la compréhension d'une œuvre d'art n'est jamais exhaustive, mais que cela ne nous empêche pas de chercher à la comprendre et à lui rendre justice. Il n'y a donc pas d' " opacité » de l'art en général, mais une difficulté plus ou moins grande à traduire les contenus et les significations des œuvres. ii) Néanmoins, 
on ne peut pas dire n'importe quoi à propos d'une œuvre ; on peut donc à tout le moins circonscrire son degré de traductibilité. Et chaque traduction verbale envisageable a des conséquences pour l'intérêt, le degré de réussite et d'importance que nous accorderons à l'œuvre. Il ne me semble donc pas que cette question trop générale de l'opacité de l'art ait une incidence sur ma thèse quant à la meilleure compréhension en relation avec des hypothèses évaluatives.

\section{2.}

Le deuxième groupe de remarques auxquelles j'aimerais répondre concerne les espoirs que je fonde sur la critique. Michel Ratté observe fort justement que je suis moi-même très sévère lorsque je parle de l'état actuel de la critique. Il évoque un peu excessivement le " cynisme » [p. 428] dont je me rendrais coupable : au moment même où je développe une théorie du débat critique, j'admets " qu'il y a de moins en moins de répondants à [mon] appel, voire de personnes en mesure de savoir de quoi [je] parle » [ibid.]. Cette contradiction, qui est presque une définition de la démarche philosophique ou de toute réflexion sur des principes, ne me semblerait guère inquiétante. Mais je ne suis pas aussi pessimiste que semble le croire Michel Ratté, et surtout, je ne vois guère de solution de rechange.

a) Je ne suis pas pessimiste, car " la critique existe ». En France, par exemple, la critique de cinéma fait preuve d'une remarquable vitalité. La preuve de son existence a été apportée, il y a quelques mois, par la révolte de certains cinéastes contre la critique de la presse française. Les cinéastes en question, rarement auteurs de chefs-d'œuvre, ont considéré que la critique ne devait pas publier d'éreintage sans avoir donné une chance à leurs films. Cet épisode, qui a rempli les colonnes des journaux pendant plusieurs mois en 1999, est significatif à plus d'un titre. Il révèle que la critique de cinéma française, contrairement à ce qui se passe fréquemment, aujourd'hui, dans le domaine de la littérature ou des arts plastiques, n'est en aucun cas réductible à la " promotion » des œuvres. Elle est indépendante et exigeante. Il se peut même qu'elle soit parfois trop exigeante et ne tienne pas compte des différents niveaux de prétention des œuvres. Dans les domaines des autres arts, dont le public est généralement moins nombreux et la part de marché d'autant plus contestée, la critique n'est pas non plus inexistante, même si elle est souvent handicapée par des déformations professionnelles de différents types : rapport trop étroit des critiques aux écrivains ou aux artistes, aux maisons d'édition et aux musées et galeries, où ils jouent le rôle de conseillers, conservateurs ou de commissaires d'exposition ; surcharge du langage critique par des jargons philosophiques en vogue, le plus souvent d'obédience nietzschéenne, heideggérienne ou post-structuraliste ; ressentiment populiste à l'égard des œuvres complexes. Souvent aussi, lorsque le succès ou l'insuccès de certaines œuvres est assuré par des pouvoirs économiques ou politiques, la critique est totalement court-circuitée. 


\section{Philosophiques / Automne 2000}

b) Par rapport à ces déformations et ces sabotages, je ne vois vraiment pas ce qui peut redresser la situation, si ce n'est une pratique revigorée de la critique à grande échelle, fondée sur une bonne connaissance de l'histoire des arts et sur un exercice du jugement dès le plus jeune âge. Je considère même que la critique n'est pas l'apanage des critiques experts : tout récepteur la pratique avec plus ou moins de finesse et de talent. Contrairement à ce que semble déduire Michel Ratté de mes remarques, je n'ai aucun mépris pour l'histoire de l'art ou pour les sciences humaines. Simplement, l'histoire de l'art ne saurait se substituer à la critique, comme elle prétend parfois le faire en légitimant les œuvres par leur seul pedigree. L'esthétique non plus ne peut guère se substituer à la critique. Son discours se situe à un niveau d'abstraction trop éloigné des œuvres ; elle a affaire aux concepts et aux méthodes employés et intervient plutôt lorsque le débat critique est dans l'impasse. Quand, sortant du cinéma, nous constatons que nos avis ne se recoupent d'aucune façon, nous nous interrogeons sur nos approches respectives : profanes ou experts, nous touchons alors à l'esthétique. Il en est de même pour les débats entre experts.

c) Michel Ratté se demande en quoi, d'après ma description, nos débats critiques se distinguent de nos débats sur les normes. Il va même jusqu'à poser cette question provocatrice : "pourquoi faire une différence entre monde social et monde subjectif » (voir sa note 22). N'ai-je pas dit qu'il faut, dans les deux cas, être participant d'un jeu commun pour comprendre les objets de ces "mondes»? Cette incompréhension de sa part est la raison pour laquelle il pense que les débats critiques, tels que je les conçois, devraient aboutir, comme les débats moraux, à des "normes contraignantes »[p. 436], chose que j'exclus formellement et qui ne vaut d'ailleurs que pour le droit. La différence entre le partage de "mondes subjectifs » et la participation à un univers social commun tient notamment au fait que l'intérêt aux œuvres d'art ne fait l'objet d'aucune obligation. S'il existe des règles du jeu dans le domaine de l'art, elles n'entrent en action que dans la mesure où une personne entre volontairement dans le jeu d'une œuvre. Celle-ci lui impose alors certains parcours et certains modes de compréhension, sans quoi le récepteur passe à côté du propos de l'œuvre. C'est pourquoi il me semble que le "relativisme » esthétique se trompe de cible. Chacun est évidemment libre d'exprimer ses préférences et de se retirer du débat critique, voire de l'ignorer. Cela n'empêche pas les interrogations sur le sens, la réussite, l'intérêt et l'importance des œuvres de se poursuivre et d'avoir des effets, y compris sur le degré de reconnaissance des artistes.

\section{3.}

La question de "l'homologation » des œuvres est, en effet, elle aussi liée à la structure paradoxalement "intersubjective » des œuvres d'art. Ratté me caricature en écrivant que "nous sommes rappelés [par moi, RR] à l'exi- 
gence intersubjective comme à une injonction idéale qui fait oublier le réel : l'arbitraire massif, par rapport aux critères de la rationalité esthétique, des motivations qui autorisent l'entrée d'œuvres dans le système de l'art " [p. 440]. C'est le contraire qui est vrai : si cet arbitraire n'existait pas, je n'aurais pas eu besoin de développer ma réflexion.

a) Si l'intersubjectivité est ici paradoxale, c'est parce qu'elle ne régit que les modalités d'accès à un univers subjectif, non l'intégration à un monde commun, comme c'est le cas de l'intersubjectivité sociale. Pour être considéré comme une œuvre d'art, un objet, performance ou texte doit présenter certaines caractéristiques, difficilement définissables, je le concède, mais qui ne sont pas arbitraires. Il s'agit notamment du fait qu'une œuvre d'art ne peut guère fonctionner si sa signification est strictement privée ; elle bascule alors vers le témoignage personnel sans enjeu artistique. Si elle est rigoureusement réductible à une information objective, elle bascule vers le document. Si elle relève d'une absence totale de savoir-faire, elle reste infra-artistique : le fait de réussir par chance une jolie photo ne fait de personne un artiste photographe, tandis que la recherche savante du brut, du non-fini ou de l'objet trouvé peut, au contraire, être considérée comme une sorte de savoir-faire. Ces règles sont à manier avec précaution; elles indiquent néanmoins que les œuvres d'art deviennent ce qu'elles sont, davantage par une " homologation » publique que par une définition subjective.

Il ne faut pas se tromper sur le sens du terme. L'erreur de Michel Ratté est d'identifier sans nuance toute forme d'homologation à une reconnaissance institutionnelle. Or, c'est exactement le contraire que je propose. Le public qui s'intéresse à une œuvre non distribuée par les principaux circuits et médias lui assure une reconnaissance et donc une homologation légitime. En revanche, il arrive aussi que des " homologations » institutionnelles œuvres de commande, favoritisme, subventions, expositions annoncées en grande pompe - rencontrent le désintérêt et l'indifférence générale, autrement dit une délégitimation parfois cruelle. L'action institutionnelle et la reconnaissance par un public sont deux processus bien distincts, même si les deux choses se recoupent fréquemment : heureusement, tous les créateurs bien diffusés ne sont pas bénéficiaires d'un favoritisme immérité. Succès n'égale pas compromission, et tout artiste marginalisé n'est pas un génie méconnu.

b) Michel Ratté a raison, cependant, d'insister sur un danger inhérent à la concentration des pouvoirs institutionnels qui caractérise notre époque. Compte tenu de l'inertie des groupes d' " experts » chargés de sélectionner les œuvres dignes de subvention, d'exposition, d'exécution ou de publication, certaines tendances qui vont à contre-courant risquent à tout instant d'être écartées, avant même qu'une " homologation » par le public ait pu se produire. Il faut donc veiller à la pluralité et à la diversité des entrées dans les circuits de diffusion, de sélection et de subvention, ce qui est une des tâches les plus importantes de la politique culturelle à l'époque de la concentration des 


\section{8 · Philosophiques / Automne 2000}

médias et des pouvoirs. Comme il ne faut pas trop se fier à la volonté décentralisatrice de ces instances, c'est aussi un sujet qui ne pourra jamais se passer d'initiatives civiques et d'engagements individuels d'artistes et d'amateurs. Sur ce plan, je fais entièrement confiance à la combativité de Michel Ratté. 